Gut, 1962, 3, 32

\title{
The results of exfoliative cytology studies in 50 patients with symptoms of large bowel disorder
}

\author{
J. I. BURN and R. A. SELLWOOD \\ From the Department of Surgery, Hammersmith Hospital and Postgraduate Medical School of London
}

SYNOPSIS The results of exfoliative cytology studies in 50 patients with symptoms of large bowel disorder are analysed. Certain cases are described in detail. Of 23 patients with cancer, a positive cytological result was obtained in nineteen. One false positive result was recorded.

Suggestions are made as to the usefulness of this investigative technique, and mention is made of the increased degree of accuracy obtained when a membrane filter is used to concentrate the cellular deposit.

The colon, perhaps more than any other organ in the body, is susceptible to benign disorders which produce symptoms and physical signs indistinguishable from those due to carcinoma. There is no specific symptom complex peculiar to malignant disease of the large bowel. Vague abdominal pain, altered bowel habit, and the passage of blood or mucus in the stools are as likely to be due to diverticulitis coli or colitis as to a large bowel neoplasm. Such symptoms always require investigation but the differentiation between carcinoma and diverticulitis in particular is a well-known problem (Thompson, 1959).

The fallibility of the available investigations adds to the difficulty. Frequently the lesion is beyond the reach of the sigmoidoscope and barium enema studies have their limitations in diagnostic accuracy. Even at laparotomy the nature of the lesion may be in doubt, and frozen section histological examination is often not practicable.

There is an accepted need of greater diagnostic accuracy, so that the extent of an operation for cancer of the large bowel can be planned beforehand. Methods designed to prevent dissemination of cancer cells at the time of surgical resection rely upon mobilization of the tumour with the minimum of handling and early ligation of blood vessels. Where doubt exists as to the nature of the lesion, this aim is frequently thwarted. Again, with the increasing use of cancer-toxic agents at the time of operation, accurate pre-knowledge of the existence of the cancer is of obvious benefit.

Recent reports on the use of exfoliative cytology in the diagnosis of cancer of the large bowel have been encouraging in the degree of accuracy demonstrated (Ebeling and Little, 1957; Raskin, Palmer, and Kirsner, 1959; Oakland, 1961). This paper records the results obtained in a further 50 patients with large bowel symptoms in whom this investigation was performed.

\section{METHODS}

The technique employed in this series has been previously described in detail (Burn, 1961), and follows closely that employed by Raskin and his colleagues (1959). Great importance was attached to the necessity of ensuring that the diagnostic washings obtained were free from all faecal and other detritus. If contamination occurs, then accurate cytodiagnosis becomes impossible. The specimens for examination were collected through a long colonic tube designed to ensure the maximum return of the diagnostic washing. This tube (Fig. 1) has an inflatable balloon which helps to retain its position in the colon, and also ensures that all the returning fluid passes through the tube for collection.

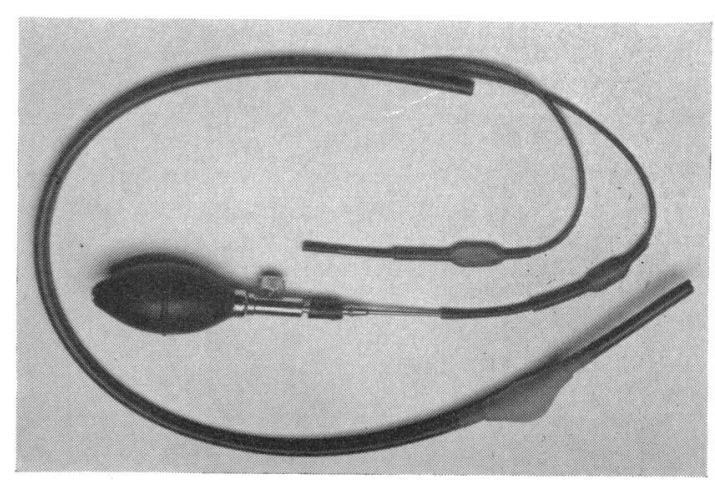

FIG. 1. Colonic tube used to collect diagnostic washings. 
In order to overcome the problem of isolating cells from a large volume of fluid the membrane filter technique was adopted in many of these patients, using a five micron Millipore filter (Cameron and Thabet, 1959; Burn, 1961; Knoernschild, Cameron, and Zollinger, 1961). This method also ensures that the majority of cells are retained for examination upon the surface of the filter. In the routine method of centrifuging the washings and examination of the sediment obtained, malignant cells may well be missed.

\section{RESULTS}

Table I summarizes the diagnoses in the 50 patients, and the results of the cytological examinations are given in Table II.

An absolute diagnosis of carcinoma of the large bowel was made in 15 of these patients by histological examination of a resected operation specimen. Seven other patients who were submitted to laparotomy were found to have carcinomas which were beyond the scope of surgical excision. In one other patient who was not operated upon, the diagnosis of

\section{TABLE I}

FINAL DIAGNOSIS IN 50 PATIENTS EXAMINED

Diagnosis $\quad \begin{aligned} & \text { Number of } \\ & \text { Patients }\end{aligned}$

Carcinoma of pelvic colon

Carcinoma of transverse colon

Carcinoma of rectum

Carcinoma of caecum

Previous operation for cancer of the colon

(a) Recurrence caecal carcinoma

(b) Recurrence carcinoma rectum

(c) No recurrence

Diverticulitis pelvic colon

Ulcerative colitis

Spastic colon

Intestinal adhesions

Carcinoma of the ovary

Retroperitoneal tumour

Peri-sigmoiditis (radiation)

Benign rectal polyp

Megacolon

Granuloma rectum

Enteritis

Appendicitis

Constipation

13
3
3
2

1
1
3
9
2
2
2
1
1
1
1
1
1
1
1
1

TABLE II

RESULTS OF CYTOLOGICAL EXAMINATION IN 50 PATIENTS

\begin{tabular}{|c|c|c|}
\hline $\begin{array}{l}\text { Patients with } \\
\text { Malignant } \\
\text { Disease of } \\
\text { Large Bowel }\end{array}$ & $\begin{array}{l}\text { Patients without } \\
\text { Malignant } \\
\text { Disease of } \\
\text { Large Bowel }\end{array}$ & $\begin{array}{l}\text { Total Number } \\
\text { of Patients } \\
\text { Examined }\end{array}$ \\
\hline
\end{tabular}

Cytology positive

for malignant 19

Cells

$(82.5 \%$ accuracy)

20

Cytology negative

for malignant $\quad 4$

cells

26

(96\% accuracy)

30

Total

23

27

50

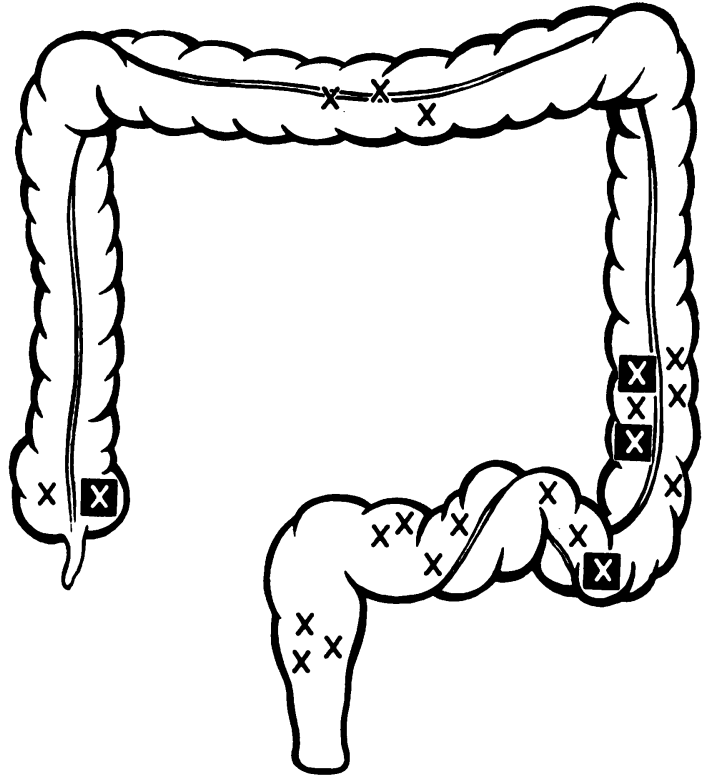

FIG. 2. The position of 21 of the malignant lesions. (The two local recurrences are not included.)

$$
{ }^{-} \mathrm{X}=\text { Cytology positive for malignant cells. }
$$

$\mathrm{X}=$ Cytology negative for malignant cells.

malignant disease was confirmed at necropsy. Thus in 23 patients an absolute diagnosis of cancer was made. The sites of the lesions are diagrammatically represented in Fig. 2. Malignant cells were identified from colonic washings in 19 of these patients, an accuracy of $82.5 \%$. Figs. 3 to 5 are examples of adenocarcinoma cells obtained from these patients.

It is interesting to observe that in the four patients with a false nega 'ive result, routine smears only were made. Where the membrane filter technique was employed, it was always possible to identify malignant cells in patients with carcinoma.

Five patients in the series had previously undergone operation for carcinoma of the colon, and were examined because of suspected local recurrence. Two were found to be positive cytologically (later confirmed at operation) and are included in the 23 patients above. One of these patients had previously undergone right hemicolectomy for carcinoma of the caecum, and the other anterior resection for carcinoma of the upper rectum. The three patients with negative cytological examination have not been submitted to further operation and remain well.

A variety of conditions accounted for the symptoms in the remaining 24 patients (Table I). Diverticulitis of the colon was the most common, but two patients were found to have malignant disease in 


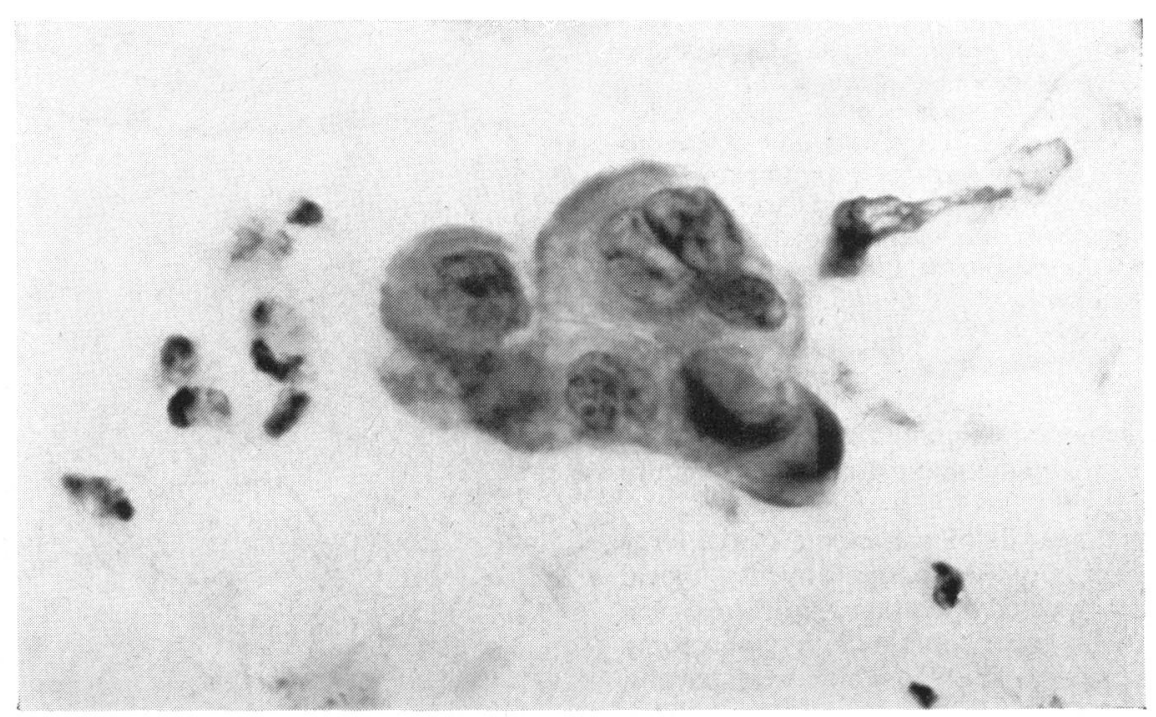

FIG. 3

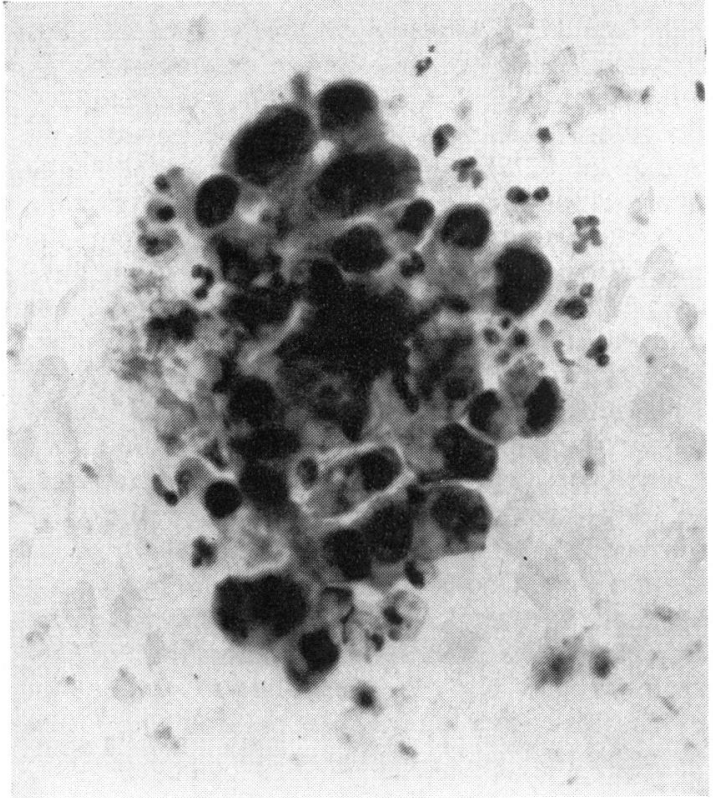

FIG. 4

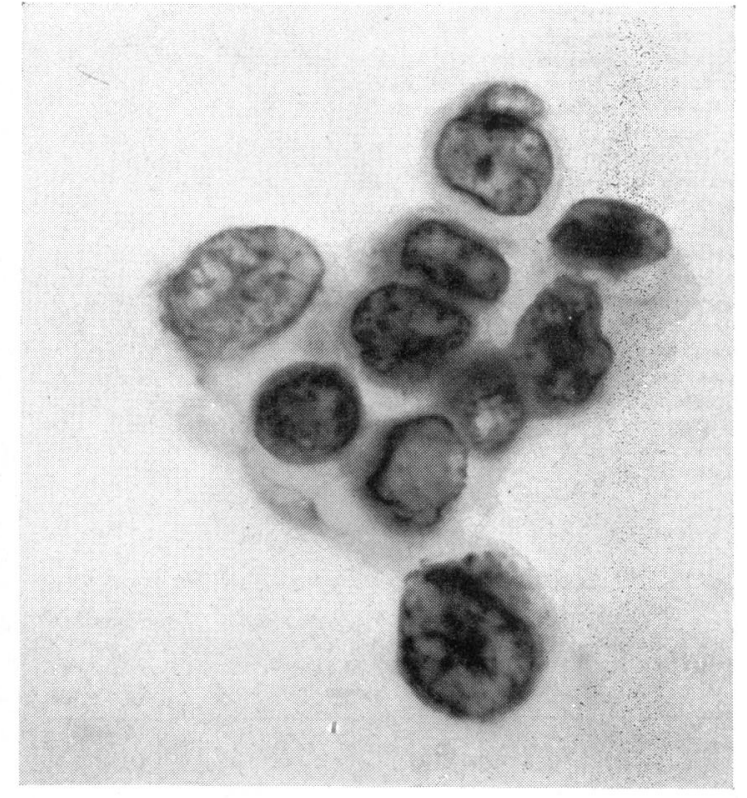

FIG. 5

FIGS. 3, 4, AND 5. Examples of malignant cells obtained from diagnostic washings in patients with carcinoma of the large bowel. (Fig. 3, $\times 1,000$, Fig. 4, $\times 500$, Fig. 5, $\times 1,260$.) We are indebted to the Royal Society of Medicine for permission to publish Fig. 5. 
the abdomen other than in the colon, one an ovarian carcinoma and the other a retroperitoneal tumour. Eleven of the patients with benign disease of the large bowel had the diagnosis confirmed at operation. Of the remainder, many have been treated by medical means and are symptom free. All have been kept under observation for at least three months, and the presence of an undetected malignant lesion is now unlikely.

One false positive result was recorded. This patient was suspected of having a malignant lesion of the sigmoid colon both clinically and on radiological examination. One clump of cells was seen from the colonic washings which was confidently thought to be malignant. At laparotomy, however, thorough exploration failed to reveal a cancer although the sigmoid colon was distorted by adhesions from a previous abdominal operation.

The recording of false positive appears elsewhere (Wisseman, Lemon, and Lawrence, 1949; Bader and Papanicolaou, 1952). It is possible that contamination from a previous patient examined was responsible in our example, as the cells may have been refrigerated within the collecting chamber due to its being inadequately cleaned after use.

We have seen a variety of cells which bear no resemblance to the normal colonic cytology but which represent damaged and degenerate epithelial cells. These sometimes give rise to difficulty in interpretation as they may have a very suspicious appearance, but we have preferred to avoid such terms as 'possibly malignant' when assessing such groups of cells. Where the characteristic features of malignancy are not present, we have labelled the result negative. Our experience in the recognition of malignant cells was increased by the examination of smears made from the surface of cancers of the large bowel immediately after resection (Fig. 6), and by the examination of washings obtained by perfusing the lumen of resected specimens with cold Ringer-Locke solution.

\section{CASE REPORTS}

CASE 1 An 82-year-old man gave the history of having noticed a lump in the abdomen for three weeks, associated with progressive constipation. Four years previously, investigation for constipation had demonstrated the presence of diverticulitis coli.

On examination the presence of a mass in the left iliac fossa was confirmed, together with evidence of recent weight loss.

A barium enema showed an obstructive lesion of the sigmoid loop, thought probably to be due to carcinoma but with diverticulitis changes in the distal loop. Sigmoidoscopy was negative.

Cytology Colonic washings were positive for malignant cells.

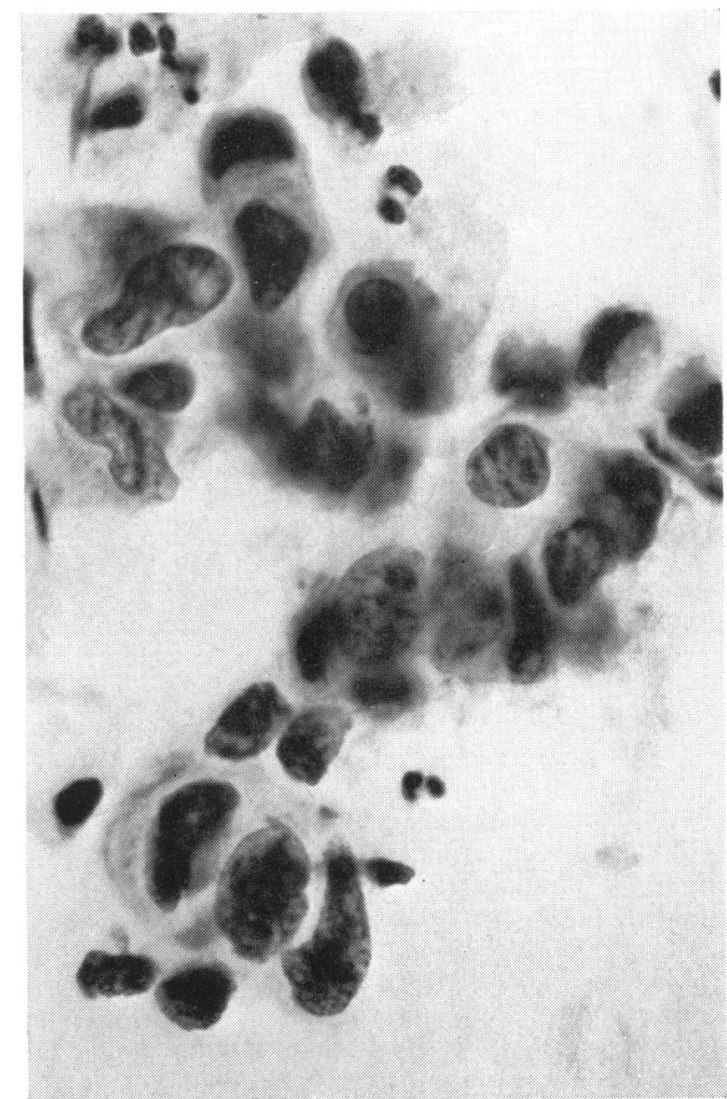

FIG. 6. Malignant cells obtained from tumour smear $(\times 920)$.

At operation, partial colectomy was performed for carcinoma of the pelvic colon.

Colonic cytology confirmed the presence of a cancer already suspected on barium enema.

CASE 2 The patient, a 47-year-old man, presented with the history of intermittent bleeding from the rectum for one week.

Physical examination was completely normal apart from slight tenderness in the right iliac fossa.

Barium enema studies demonstrated no abnormality, but a raised ulcerated lesion was seen on sigmoidoscopy at $16 \mathrm{~cm}$. Biopsy report was 'well differentiated adenocarcinoma'.

Cytology Colonic washings were positive for malignant cells. At operation, anterior resection of the carcinoma was performed.

Barium enema studies failed to demonstrate this lesion which was diagnosed by sigmoidoscopy. A 
large number of malignant cells was recovered from colonic washings in this patient.

CASE 3 An 80-year-old woman was investigated for an eight-month history of lower abdominal pain and increasing constipation. There was tenderness in the left iliac fossa on examination, with a doubtful mass in the pouch of Douglas.

Barium enema examination showed well-marked diverticulosis with diverticulitis of the pelvic colon. The lumen of the bowel was irregular, and carcinoma could not be excluded. Sigmoidoscopy was normal as far as the instrument could be passed.

Cytology Cytological examination in this patient was negative for malignant cells, but large numbers of inflammatory cells were present with increased exfoliation of normal epithelial cells.

At operation, the appearances were those of diverticulitis and sigmoid colectomy was performed. Histological examination of the specimen showed no evidence of malignancy.

Carcinoma was excluded by cytological examination, and this opinion was confirmed at operation.

CASE 4 A 66-year-old woman was admitted to hospital with a four-month history of malaise, abdominal discomfort, and increasing abdominal distension. Physical examination demonstrated marked ascites and a hard mass in the upper abdomen.

Barium studies were not carried out in this patient, and sigmoidoscopy was normal.

Cytology Colonic washings were positive for malignant cells. Examination of the ascitic fluid also demonstrated the presence of cancer cells. A vaginal smear examination was negative.

Laparotomy confirmed the presence of an extensive carcinoma of the transverse colon with widespread peritoneal and hepatic metastases. The growth was beyond surgical removal and the patient died soon after operation.

The cytological examination confirmed the clinical suspicion of carcinoma of the transverse colon.

CASE 5 A 66-year-old man gave a two-year history of lower abdominal discomfort and rectal bleeding. His general health was poor, as he suffered from severe angina pectoris and generalized arthritis. Examination of the abdomen demonstrated tenderness in the left iliac fossa. Rectal examination was normal.

A barium enema showed extensive diverticulosis and sigmoidoscopy suggested the presence of diverticulitis in the lower pelvic colon.

Cytology Colonic washings were negative for malignant cells.

No operation was performed on this patient, and he was treated by dietary and other medical measures. Apart from an occasional exacerbation his symptoms have been well controlled on this régime for 10 months with nothing to suggest underlying malignant disease.
A negative cytological report assisted in making the decision to persevere with medical treatment in this patient, who was an obviously undesirable candidate for surgery.

CASE 6 The patient, a 72-year-old man, complained of episodes of colicky abdominal pain and increasing constipation. He had undergone right hemicolectomy for a well-differentiated adenocarcinoma of the caecum 18 months previously. Physical examination revealed some abdominal distension and tenderness. Rectal examination was normal.

A barium enema was reported as being normal apart from a few diverticula in the sigmoid loop. There was no evidence of recurrence of tumour. The faeces were consistently positive for occult blood.

Cytology Colonic washings were positive for malignant cells, together with many inflammatory cells.

At operation, a large local recurrence of the carcinoma was present in the region of the anastomosis, extending into the posterior abdominal wall and beyond the scope of surgical removal. There was evidence of partial obstruction with dilatation of small bowel loops. By-pass ileo-transverse colostomy was performed. The patient died from a coronary thrombosis soon after operation.

The diagnosis of recurrence of the cancer was made on cytological examination in this patient, confirming the clinical impression.

CASE 7 A 75-year-old man was investigated for an eight-month history of loose motions, cramp-like abdominal pain, and the passage of mucus through the rectum. Physical examination was negative apart from slight, but definite tenderness in the left iliac fossa.

Diverticulitis of the sigmoid loop was demonstrated on radiological examination. Sigmoidoscopic examination was normal for $20 \mathrm{~cm}$. but examination of the stools was consistently positive for occult blood.

Cytology Cytological studies were negative for malignant cells, but there were numerous inflammatory cells and an increased number of exfoliated normal epithelial cells.

The patient was put on a dietary and medical régime with an apparent improvement in his condition for one month. The symptoms then re-appeared with increasing severity and he was submitted to laparotomy. At operation, the presence of diverticulitis was confirmed, and sigmoid colectomy was performed. Histology of the resected specimen demonstrated the presence of a small, well-differentiated adenocarcinoma.

This chastening experience illustrates one of the pitfalls of diagnosis in cancer of the large bowel.

\section{DISCUSSION}

In assessing the value of a new investigative technique, it is necessary to examine carefully the accuracy of the already available means of diagnosis. 
In the 23 patients with carcinoma, biopsy at sigmoidoscopy was positive for the cancer in nine patients. That is to say 14 had lesions which were either beyond the range of the instrument or the biopsy was inaccurately taken. Barium enema examination was performed in $\mathbf{4 0}$ of the $\mathbf{5 0}$ patients in the series. In these 40 patients, an accurate diagnosis was made in 31 . In the 23 patients with carcinoma of the large bowel, barium enema examination was made in 16, an accurate diagnosis of cancer being made in 11 . Frequently the examination suggested the presence of a malignant lesion, but the appearances were such that alternative diagnoses were also suggested and doubt existed. That this doubt may be continued even at operation is demonstrated by Case 7 .

In our hands, cytological examination was found wanting in four of the patients with carcinoma. It is unlikely that these four cancers failed to exfoliate cells into the lumen of the bowel and therefore this failure must be attributed to errors in technique and lack of experience in the recognition of malignant cells. The presence of coincidental inflammatory conditions and secondary infection of the cancer makes the identification of the cells more difficult, owing to the large number of pus cells present and the rapid degeneration of the malignant cells. Also, it must be accepted that collection of cells from the left side of the colon by the diagnostic washing technique will always be more reliable than from the right side of the colon.

It has been suggested that there is an increased exfoliation of normal colonic epithelial cells where cancer exists. This has not been an invariable finding in our experience, and indeed we found this to be so more frequently in the presence of inflammatory lesions than in cancer. Patients with diverticulitis and ulcerative colitis demonstrated a high rate of exfoliation.
Our impression after using the investigation in a relatively small series of patients is that it could become a valuable adjunct to the methods of investigation already available. If considered together with the history, clinical findings, radiological examination, and sigmoidoscopy, a high degree of diagnostic accuracy should be possible before the patient is subjected to operation.

We are grateful to Professor Ian Aird for his help in preparing this paper, and thank all the members of the surgical staff of the Hammersmith Hospital for permission to carry out this investigation on patients under their care.

The colonic tube used for the diagnostic washings was kindly supplied by Franklin's Rubber Company, Dalston.

\section{REFERENCES}

Bader, G. M., and Papanicolaou, G. N. (1952). The application of cytology in the diagnosis of cancer of the rectum, sigmoid and descending colon. Cancer, 5, 307-314.

Burn, J. I. (1961). Exfoliative cytology of the colon. Proc. roy. Soc. Med., 54, 726-729.

Cameron, A. B., and Thabet, R. J. (1959). Recovery of malignan cells from enema returns in carcinoma of the colon. Surg. Forum, 10, 30-33.

Ebeling, C. E., and Little, J. W. (1957). The demonstration of malignant cells exfoliated from the proximal colon. Ann intern. Med., 46, $21-29$.

Knoernschild, H. E., Cameron, A. B., and Zollinger, R. M. (1961). Millipore filtration of colonic washings in malignant lesions of the large bowel. Amer. J. Surg., 101, 20-22.

Oakland, D. J. (1961). The diagnosis of carcinoma of the large bowel by exfoliative cytology. Brit. J. Surg., 48, 353-362.

Raskin, H. F., Palmer, W. L., and Kirsner, J. B. (1959). Exfoliative cytology in diagnosis of cancer of the colon. Dis. Colon Rect., 2, 46-57.

Thompson, H. R. (1959). Diverticulitis of the Colon. Postgrad. med. $J ., 35,86-91$.

Wisseman, C. L. Jr., Lemon, H. M., and Lawrence, K. P. (1949) Cytologic diagnosis of cancer of the descending colon and rectum. Surg. Gynec. Obstet., 89, 24-30. 